\title{
Cordão umbilical equino: características na gestação e avaliação no pós-parto
}

\author{
Equine umbilical cord: features during gestation and postpartum evaluation \\ Cordón umbilical equino: características durante la gestación y la evaluación posparto
}

Recebido: 08/01/2021 | Revisado: 10/01/2021 | Aceito: 11/01/2021 | Publicado: 13/01/2021

\author{
Gabriela Castro da Silva \\ ORCID: https://orcid.org/0000-0002-8555-2140 \\ Universidade Federal de Pelotas, Brasil \\ E-mail: gabicastrovini@gmail.com \\ Carlos Eduardo Wayne Nogueira \\ ORCID: https://orcid.org/0000-0002-8555-7953 \\ Universidade Federal de Pelotas, Brasil \\ E-mail: cewn@terra.com.br \\ Fernanda Maria Pazinato \\ ORCID: https://orcid.org/0000-0002-3616-1274 \\ Universidade do Oeste de Santa Catarina, Brasil \\ E-mail: Fernanda.pazinato@unoesc.edu.br \\ Tais Del Pino \\ ORCID: https://orcid.org/0000-0003-4583-8280 \\ Universidade Federal de Pelotas, Brasil \\ E-mail: tais.pino@gmail.com \\ Rafaela Bastos Silva \\ ORCID: https://orcid.org/0000-0003-0456-3284 \\ Universidade Federal de Pelotas, Brasil \\ E-mail: rafaelaaa.bastos@gmail.com \\ Bruna da Rosa Curcio \\ ORCID: https://orcid.org/0000-0001-8678-3816 \\ Universidade Federal de Pelotas, Brasil \\ E-mail: curciobruna@hotmail.com
}

\begin{abstract}
Resumo
No equino, assim como nos mamíferos em geral, o cordão umbilical é a única fonte de condução de nutrientes, gases e metabólitos da placenta para o feto, e o seu adequado desenvolvimento é de extrema importância para a saúde fetal durante a gestação. O objetivo da presente revisão é caracterizar o cordão umbilical durante a gestação em equinos, bem como descrever as principais alterações e achados casuais na avaliação deste junto à placenta e o neonato no pósparto. Como metodologia foi realizada uma revisão de literatura qualitativa sobre as características do cordão umbilical na espécie equina utilizando artigos disponíveis nas plataformas Mendeley, MEDLINE, PubMed e SciELO. Como resultados podemos identificar as características de anatomia e desenvolvimento. Assim como as alterações mais comuns: presença de cordões excessivamente longos (apresentando mais de $85 \mathrm{~cm}$ de comprimento ao nascimento), torção do cordão e alterações do úraco. Estas podem ser de origem não infecciosa (hérnias umbilicais e o úraco persistente) ou infecciosa (onfalopatias). Como achados casuais podemos citar as placas amnióticas e a ossificação dos remanescentes do cordão umbilical e saco vitelínico. Conclui-se que a avaliação do cordão umbilical no pós-parto imediato auxilia no reconhecimento de alterações não observadas durante a gestação, sendo imprescindível caracterizar as alterações de relevância clínica dos achados casuais na avaliação do cordão umbilical em equinos.
\end{abstract}

Palavras-chave: Obstetrícia; Éguas; Placenta; Saco vitelínico.

\begin{abstract}
In equines, as other mammalian species, the umbilical cord is the only source of conduction of nutrients, gases, and metabolites from the placenta to the fetus, and its adequate development is super important for fetal health during pregnancy. The aim of the present review is to characterize the umbilical cord during pregnancy in horses, as well as to describe the main changes and casual findings in its evaluation with the placenta and the newborn in the postpartum period. For methodology a qualitative literature review was performed about the characteristics of the umbilical cord in the equine specie using articles selected using Mendeley, MEDLINE, PubMed and SciELO platforms. As a result, we can identify the characteristics of anatomy and development. Moreover, the most common changes in the umbilical cord are the identification of excessive length cords (over $85 \mathrm{~cm}$ at term), pathological twisting, or torsion of the cord and changes of uracus. These can be of non-infectious (umbilical hernias and patent uracus) or infectious conditions (omphalopathies). As casual findings, we can find amniotic plaques and ossification of remnants of the umbilical cord and yolk sac. We conclude that the evaluation of the umbilical cord in the immediate postpartum
\end{abstract}


period could help in the recognition of changes not observed during pregnancy, and it is essential to characterize the changes of clinical relevance of the casual findings in evaluation of the umbilical cord in equines.

Keywords: Obstetrics; Mares; Placenta; Yolk sac.

\section{Resumen}

En el caballo, así como en los mamíferos en general, el cordón umbilical es la única fuente de conducción de nutrientes, gases y metabolitos de la placenta al feto, y su adecuado desarrollo es de suma importancia para la salud fetal durante la gestación. El objetivo de la presente revisión es caracterizar el cordón umbilical durante la gestación en caballos, así como describir los principales cambios y hallazgos casuales en su evaluación con la placenta y el recién nacido en el posparto. Como metodología, se realizó una revisión bibliográfica cualitativa sobre las características del cordón umbilical en la especie equina utilizando artículos disponibles en las plataformas Mendeley, MEDLINE, PubMed y SciELO. Como resultado, podemos identificar las características de la anatomía y el desarrollo. Así como, los cambios más frecuentes en el cordón umbilical: la identificación de cordones excesivamente largos (más de $85 \mathrm{~cm}$ de longitud al nacer), torsión del cordón y cambios en el uraco. Estos pueden ser de origen no infeccioso (hernias umbilicales y uraco persistente) o infecciosos (onfalopatías). Como hallazgos casuales podemos mencionar las placas amnióticas y la osificación de los restos del cordón umbilical y del saco vitelino. Se concluye que la evaluación del cordón umbilical en el posparto inmediato ayuda en el reconocimiento de cambios no observados durante la gestación, y es fundamental caracterizar la relevancia clínica de los cambios de los hallazgos casuales en la evaluación del cordón umbilical en equinos.

Palabras clave: Obstetricia; Yeguas; Placenta; Saco vitelino.

\section{Introdução}

Nos mamíferos, o cordão umbilical é a rota de carreamento de sangue oxigenado e nutrientes para o feto durante a gestação, além de ser responsável por todas as trocas gasosas e metabólicas, necessárias para o desenvolvimento fetal (Wilsher et al., 2011).

O pulmão fetal não é funcional no útero, dessa forma, são os vasos umbilicais os responsáveis por suprir o feto de sangue oxigenado. Nos equinos este passa pelo ducto venoso, o maior vaso da placenta, passando pelo fígado embrionário, e deste para veia cava caudal (Mcgeady et al., 2006).

Como única fonte de condução de nutrientes, gases e metabólitos da placenta ao feto, o adequado desenvolvimento e sanidade do cordão umbilical é de extrema importância para a saúde fetal durante a gestação (Wilsher et al., 2011). As alterações de torção ou enrolamento anormal do cordão umbilical são as mais frequentemente descritas em equinos e outros mamíferos, incluindo humanos mesmo com todo auxílio e cuidados pré natais a fim de evitar os fatores de risco ao nascimento (Pieszak et al., 2020). Sendo humanos a torção de cordão umbilical pode estar associada com a trombose de vasos da face coriônica da placenta, trombose venosa umbilical, e estenose do cordão. Assim, quando a torção umbilical ocorre de forma parcial pode ser considerada com "um estado crônico", o qual promove retardo no crescimento fetal, assim como em casos de a torção umbilical ser de maior grau, ou seja, "torção aguda", ocorre a morte fetal e aborto (Machin et al., 2000).

O objetivo deste estudo é realizar uma revisão sobre características do cordão umbilical durante a gestação em equinos, bem como descrever as principais alterações e achados casuais na avaliação deste junto à placenta e o neonato no pósparto.

\section{Metodologia}

O presente estudo consta de uma revisão de literatura qualitativa como ferramenta de metodologia de pesquisa científica (Pereira et al., 2018), realizando assim uma reflexão sobre a manifestação de ideias e sua representação sobre as características do cordão umbilical na espécie equina. Para realização da revisão foram selecionados artigos utilizando as plataformas Mendeley, MEDLINE, PubMed e SciELO. Devido ao escasso número de publicações específicas na espécie equina, foram utilizados artigos publicados a partir do ano de 1975. 


\section{Anatomia e Desenvolvimento}

No equino, o cordão desenvolve-se até o $50^{\circ}$ dia de gestação, a partir da convergência das duas membranas fetais: o âmnio e o alantoide (Wilsher et al., 2011). Com a expansão do alantoide e regressão do saco vitelínico durante o desenvolvimento embrionário, o cordão umbilical aumenta gradativamente seu comprimento. Como resultado, o úraco também se alonga (Whitwell,1975).

Durante a regressão do saco vitelínico o mesmo se adere na porção média do cordão, local de projeção da membrana amniótica. A posição de regressão do saco vitelínico para o cordão constitui o sítio de ligação do alantocorion ao cordão durante toda a gestação, identificando o corno uterino e local onde a fixação do embrião ocorreu (Mariela et al., 2018).

O cordão umbilical equino é composto por três vasos umbilicais, sendo duas artérias, uma veia, e o úraco. As artérias umbilicais são oriundas das artérias ilíacas internas e a veia umbilical, que se forma da união de duas ou três veias tributárias oriundas da placenta (Carvalho et al., 2001).

A porção proximal amniótica do cordão é coberta pelo âmnio e está ligada ao feto através do umbigo, e a porção alantóica coberta pelo alantoide, está ligada ao alantocórion. A porção amniótica do cordão umbilical contém duas artérias, uma veia umbilical, e o úraco (Wilsher et al, 2011).

Na porção alantóica do cordão, ocorre maior divergência das duas artérias e múltiplas ramificações das mesmas em direção ao córion placentário. As veias e o ducto umbilical que retornam sangue para o feto tendem a estar muito próximas à estas artérias. As veias maiores unem-se na porção proximal ao final da região alantóica ou já dentro da cavidade amniótica (Wilsher et al., 2011).

Na porção distal intra-alantóide as duas artérias umbilicais principais tornam-se divergentes e multibranificado em direção ao córion e as duas veias umbilicais permanecem próximas a essas artérias. As duas veias principais se unem na extremidade proximal da porção alantóide ou apenas dentro da cavidade amniótica. O úraco conecta a bexiga urinária fetal à cavidade alantóide dentro do tecido estromal na porção amniótica (Schlafer, 2004).

Em estudo realizado em fetos equinos sem raça definida, foi observado que em entre os vasos umbilicais formam-se vasos funiculares anastomósticos, sendo essas anastomoses arteriovenosas. Em nenhuma delas houve ramos funiculares oriundos destas anastomoses. Na observação do funículo umbilical composto pelo ducto alantóíde, juntamente com os vasos, encontra-se entremeado pela gelatina de Wharton e rodeados pelo âmnio (Carvalho et al., 2001).

Visualmente o cordão umbilical normal apresenta espirais anatômicas, que não devem ser confundidas com quadros de torção. O comprimento do cordão em fetos equinos equivale geralmente ao seu comprimento corporal, variando de 28 a 70 cm em fetos com idades de 73 a 249 dias de gestação. Em criatórios de equinos PSI no sul do Brasil, a média de comprimento do cordão observada foi de 47,6 cm, com mínimo e máximo, de 30 e $84 \mathrm{~cm}$ respectivamente (Pazinato et al., 2016).

A convergência das artérias umbilicais, diretamente do alantocórion até a junção para projeção do cordão, determina o tipo de formação vascular placentária. Há três tipos principais de formação vascular resultante da convergência das artérias para o cordão na espécie equina (Whitwell, 1982).

O tipo I é o mais comumente encontrado, no qual uma artéria converge do corno gravídico, enquanto as outras estão distribuídas no corpo e corno não gravídico da placenta (Figura 1, arquivo pessoal) (Rossdale \& Ricketts, 2002). O tipo II perfaz de 20 a $23 \%$ de incidência e apresenta uma artéria saindo do corno gravídico e corpo uterino, enquanto as outras convergem do corno não gravídico (Figura 2, arquivo pessoal) (Rossdale \& Ricketts, 2002). Já o tipo III é o mais raro, no qual uma artéria converge do corno gravídico e não gravídico, e da maior parte do corpo do útero, e outra se prolonga da curvatura dorsal do corpo uterino (Rossdale \& Ricketts, 2002). O tipo III foi previamente descrito como resultante de quadros gemelares onde há redução manual de um dos gêmeos, porem o nível de incidência nestes quadros é variado. Outras variações de 
distribuição vascular na placenta foram observadas, porém sua importância sobre o desenvolvimento e sanidade fetal não são conhecidas (Whitwell, 1975).

Figura 1. Formação vascular placentária Tipo I, demonstrando projeção de uma artéria para corno gravídico (seta), e as outras convergindo para corpo e corno não gravídico (cabeça de seta). Figura 2. Tipo II de vasculatura placentária, demonstrando uma artéria convergindo do corno gravídico e corpo uterino (seta), enquanto as outras convergem
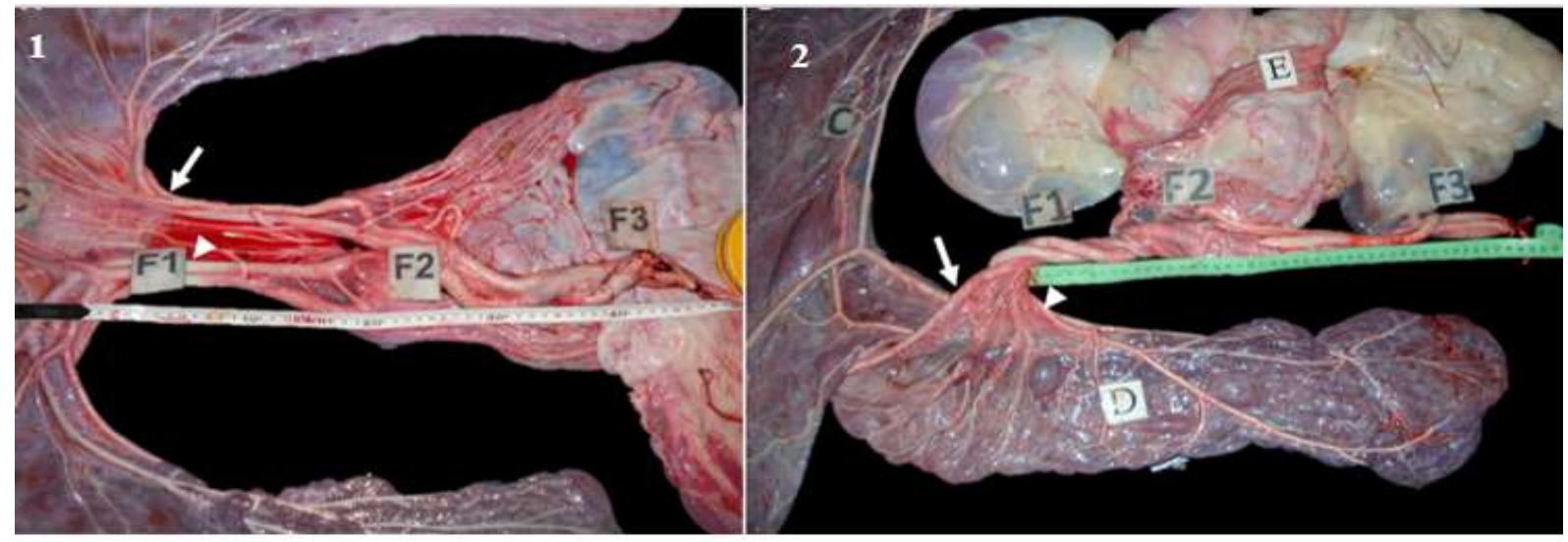

Fonte: Arquivo pessoal.

Nas figuras acima observamos os diferentes tipos de formação vascular placentária Tipo I e Tipo II, os mais frequentemente encontrados na avaliação pós-parto. Na Figura podemos identificar a projeção de uma artéria para corno gravídico, e as outras artérias convergindo para a região da placenta referente ao corpo do útero e o corno não gravídico caracterizando deste modo vasculatura Tipo I. Na Figura 2 é possível observar a diferença em relação a convergência das artérias umbilicais, sendo uma artéria convergindo do corno gravídico e corpo uterino, enquanto as outras convergem para o corno não gravídico, caracterizando a vasculatura placentária Tipo II.

\section{Alterações no Cordão Umbilical}

As maiores perdas durante a gestação são decorrentes de alterações de origem infecciosa, comprometendo a membrana corioalantóide e resultando em abortos, partos prematuros ou nascimento de neonatos não viáveis (Hong et al., 1993; Le Blanc et al., 2004). Entretanto, as anomalias de cordão umbilical têm sido descritas como a principal causa de abortos de origem não infecciosa, sendo descrito de $16 \%$ a 60,5\% de perdas gestacionais em equinos na Europa e Estados Unidos (Laugier et al., 2011; Snider, 2007; Smith et al., 2008). Em humanos, a torção do cordão umbilical ocorre em cerca de $23 \%$ e 37\% das gestações (Bencharif et al., 2010).

As lesões observadas nesses quadros incluíram torção excessiva, com edema e hemorragia abundante, áreas de estenose, dilatação de úraco e/ou veia umbilical. A identificação de hemorragia e desgarro da túnica íntima vascular, são indicativos de torção excessiva ou mesmo tração intra-uterina ou durante o parto. O feto pode muitas vezes apresentar-se autolisado, devido a expulsão ocorrer várias horas após a morte. Por vezes, o cordão umbilical pode estar enrolado em um apêndice fetal, obstruindo o fluxo sanguíneo e cursando com o aborto (Snider, 2007).

Em estudo de Whitwell. (1975), na Raça Puro Sangue Inglês, salienta que muitas mortes fetais de causa desconhecida entre 7 e 10 meses de gestação coincidem com os cordões com mais de $80 \mathrm{~cm}$, o que sugere que esses abortos poderiam estar ligados a anormalidades não diagnosticados de cordão umbilical. 
Cordões excessivamente longos podem ser um fator predisponente para problemas circulatórios e, consequentemente, causar aborto (Laugier et al., 2011), sendo que fetos que apresentaram cordões com comprimento acima de $80 \mathrm{~cm}$, apresentam risco três vezes maior de apresentar torção (Whitwell, 1975).

Além da torção e risco de aborto, cordões excessivamente longos estão associados a mudanças degenerativas nos microcotilédones e insuficiência placentária, que podem levar ao comprometimento do crescimento fetal (Frazer, 2007). Embora isso obviamente, tenha influência sobre a saúde fetal, nenhum estudo foi publicado para avaliar os efeitos sobre o crescimento ou o desempenho neonatal e/ou adulto de fetos que sobreviveram.

Quadros de implantação do cordão umbilical no corno não gestante estão relacionados a cordões umbilicais longos (Whitehead et al., 2005 apud Chenier, 2011). O mecanismo associado a essa situação não está bem esclarecido. Sugere-se que esta circunstância pode ser devido ao cordão longo permitir a migração do feto para o corno oposto durante a gestação, ou inversamente, o cordão longo se desenvolve em consequência à cinética fetal, enquanto o feto é transportado para o corno oposto (Chenier, 2011).

A espiral anatômica normal deve ser diferenciada de torção do cordão, que é patológica e provoca restrição do fluxo de sangue. Uma leve torção do cordão pode não ter efeito prejudicial detectável, entretanto ao atingir um certo grau, como $180^{\circ} \mathrm{C}$ ou acima, leva ao estrangulamento com restrição de fluxo, e consequentemente pode causar sofrimento fetal agudo (com morte fetal e aborto), ou subagudo (com compressão sanguínea e insuficiência placentária crônica resultante). Torção do segmento amniótico do cordão pode levar a constrição do fluxo de urina através úraco, resultando em distensão da bexiga (Schlafer, 2004).

Edema e hemorragia estão presentes em torções excessivas, mas em casos com apenas algumas voltas, as alterações no cordão podem ser mínimas ou sem manifestação evidente (Schlafer, 2004).

\section{Alterações do Funículo Umbilical no Neonato}

A ruptura do cordão umbilical em fetos normais ocorre no máximo $10 \mathrm{~cm}$ de distância da parede abdominal. Entretanto, em neonatos prematuros, imaturos ou mesmo que apresentem alterações nessa região, podem ocorrer sangramentos excessivos ou resultar em separação anormal favorecendo entrada de agentes infecciosos, e consequente alterações nesse remanescente do funículo umbilical (Allen et al., 2002).

Os distúrbios encontrados nos remanescentes umbilicais podem ser divididos de acordo com a sua causa, diferenciando-se entre infecciosas como a onfalite ou onfaloflebite, e não infecciosas, que incluem o úraco persistente e hérnia umbilical (White \& Huff, 1998).

Após a ruptura do cordão umbilical no momento do parto, seus remanescentes podem se tornam uma porta de entrada para agentes patogênicos, nos casos em que os cuidados com a limpeza local não são adequados (Adams, 1990).

As onfalopatias são caracterizadas por processos extra abdominais, chamados de onfalites, nas quais o tronco umbilical remanescente é acometido, e de acordo com a estrutura acometida, pode ser classificado como onfaloflebite, onfaloarterite, onfalouraquite e suas combinações (Sturion et al., 2013).

Nas enfermidades não infecciosas, a hérnia umbilical é a segunda patologia congênita mais comum em neonatos, ocasionada pelo fechamento incompleto da parede ao redor do umbigo, causando uma aposição do peritônio na fáscia e pele. Fatores como a tração excessiva do cordão, infecções umbilicais e o rompimento manual do cordão umbilical predispõem o desenvolvimento de hérnias (Nyberg et al., 2002).

Hérnias umbilicais são facilmente reconhecidas através da inspeção e palpação do local. Em sua maioria as hérnias umbilicais são pequenas, indolores a palpação e podem se fechar espontaneamente (até seis meses) sem necessidade de intervenção. Entretanto, as mais extensas podem apresentar um risco maior de complicações como estrangulamento e 
encarceramento de alças intestinais e abcesso umbilical, e por isso deve-se avaliar a necessidade de tratamento cirúrgico (Orsini, 1997).

O úraco é a comunicação fisiológica entre a bexiga e alantocórion durante a vida fetal, permitindo que a urina passe para cavidade alantoideana (Madigam \& House, 2002). Após a ruptura do cordão umbilical, o úraco se fecha e o fluxo de urina é interrompido nas primeiras 24 horas de vida do potro. A principal alteração do funículo umbilical é o "úraco persistente" que consiste na falha do fechamento dessa estrutura nesse período de 24 horas ou quando ocorre a reabertura do úraco dias após o nascimento (Stoneham \& Munroe, 2013). Os sinais clínicos mais evidentes são o gotejamento de urina e inchaço ao redor do umbigo, o qual ocorre por ação irritativa da própria urina nessa região, ou pode ser sugestivo de um processo infeccioso nessa região. A persistência do úraco é um distúrbio que pode ocorrer de forma congênita associada a quadros de imaturidade neonatal ou secundária a casos de septicemia neonatal (Knottenbelt et al., 2004).

O diagnóstico é feito com maior frequência clinicamente, a partir da visualização do sinal clínico do gotejamento ou vazamento de urina pelo tronco umbilical, esporadicamente ou durante a micção. O qual pode ser confirmado no exame ultrassonográfico da região (Paradis, 2006).

$\mathrm{Na}$ maioria dos casos ocorre a oclusão e regressão espontânea do úraco persistente somente com a limpeza e desinfecção, por imersão do coto umbilical em soluções a base de iodo (Mcauliffe \& Knottenbelt, 2014). Entretanto, se mesmo após a imersão não houver resolução do quadro, é recomendado o encaminhamento cirúrgico (Knottenbel et al., 2004).

\section{Achados Casuais}

Um dos achados mais peculiares na espécie equina é a calcificação dos remanescentes do saco vitelino, geralmente associada à hiperplasia e hipertrofia dos tecidos adjacentes (Figura 3, arquivo pessoal). Em geral são estruturas pequenas, porém podem aparecer como grandes massas pedunculares ligadas ao segmento alantóide do cordão umbilical por uma haste de tecido (Whitwell,1975).

As estruturas de saco vitelínico calcificadas podem ser erroneamente diagnosticadas como sendo um feto gêmeo mumificado. Isso provavelmente devido duas razões; a primeira devido a eventual similaridade com o amorphus globosus que ocorre na espécie bovina (anomalia fetal do desenvolvimento que ocorre em gestações múltiplas em bovinos). E a segunda razão devido a aparência macroscópica do remanescente do saco vitelino ossificado equino possuir o formato esférico com placas calcificadas na superfície externa e repletas de fluido, o que pode assemelhar-se a uma cabeça malformada (Schlafer,2004).

A ocorrência de ossificação do saco vitelínico ocorre somente na espécie equina, sendo relevantes somente nos casos que promovem interferência mecânica no fluxo de sangue do cordão umbilical para a placenta, o que pode levar a hipóxia fetal e provocar aborto (Schlafer, 2004). 
Figura 3. Presença de remanescente de saco vitelínico em porção média de cordão umbilical, demonstrando forma esférica.

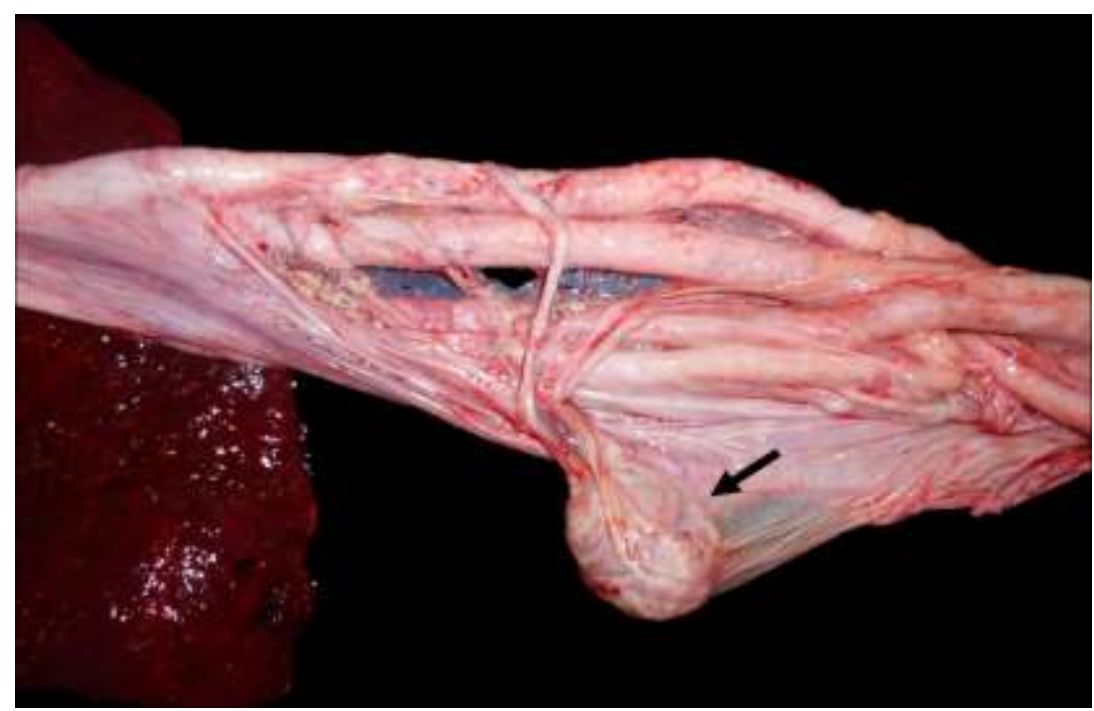

Fonte: Arquivo pessoal

Na Figura 3 é evidenciada a presença de uma massa esférica e pendular (seta) na porção média do cordão umbilical 1, que caracteriza o remanescente de saco vitelínico. Sendo esse um achado um achado casual da avaliação do cordão umbilical equino no pós-parto imediato.

Áreas de edema próximo ao local de ruptura do cordão e na região de inserção do mesmo na membrana amniótica são achados casuais comuns. Casos de alterações patológicas, como em torções graves, as áreas de edema frequentemente são acompanhadas de hemorragias e/ou petéquias locais.

No cordão umbilical também podem estar presentes placas amnióticas (Figura 4, arquivo pessoal), caracterizadas por pequenas placas queratinizadas (1 a 3mm) com aspecto de grumos, dispersas uniformemente e que se projetam ligeiramente para superfície externa, porém não apresentam uma importância funcional descrita. As mesmas são visualizadas principalmente na superfície interna do âmnio e na superfície externa do segmento amniótico do cordão umbilical, local de inserção da porção amniótica. Microscopicamente são compostas de epitélio amniótico que sofreu metaplasia escamosa (Schlafer, 2004).

Figura 4. Placas amnióticas, presentes na porção amniótica do cordão umbilical.

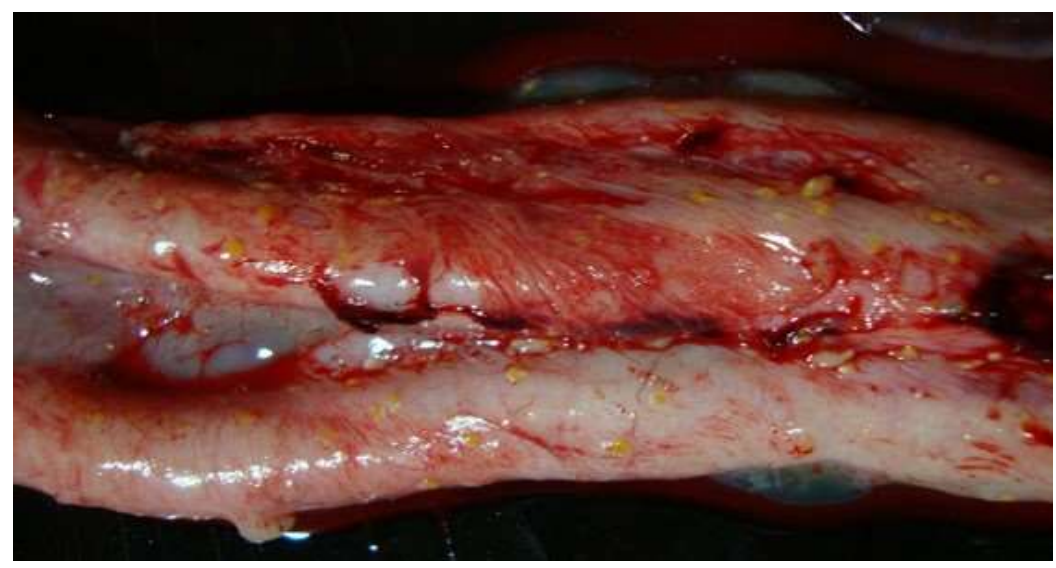

Fonte: Arquivo Pessoal. 
Na Figura 4 observamos o cordão umbilical coberto por placas amnióticas queratinizadas, um achado casual que pode ser observado na avaliação pós-parto. As mesmas são visualizadas nestes pequenos grumos amarelados se projetando na superfície externa do segmento amoniótico do cordão.

\section{Conclusão}

A avaliação do cordão umbilical no pós-parto imediato é importante para auxiliar no reconhecimento das características morfológicas e alterações não observadas durante a gestação. É imprescindível saber diferenciar achados casuais de alterações que possuem relevância clínica durante a avaliação do cordão umbilical em equinos.

Estudos futuros são necessários para a identificação e descrição detalhada das diferenças morfológicas do cordão umbilical equino em relação as particularidades de cada raça.

\section{Referências}

Adams, S. B. Urachal and umbilical dissease. (1990). Equine Clinical Neonatology, Philadelphia.

Allen, W. R., Wilsher, S., Turnbull, C., Stewart, F., Ousey, J., Rossdale, P. D. \& Fowden, A. L. (2002) Influence of maternal size on placental, fetal and postnatal growth in the horse. I. Development in utero. Reproduction 123, 445-453.

Bencharif, D. (2010). Case Study of Abortion in a Mare: Coelosomy with urachal dilatation following umbilical cord torsion. Journal of Equine Veterinary Science, 30(4), 208-212.

Carvalho, F. R., Miglino, M. A., Severino, R. S., Ferreira, F. A., \& Santos, T. C. (2001). Aspectos morfológicos do funículo umbilical em equinos (Equus caballus, Linnaeus, 1758). Brazilian Journal Veterinary Research and Animal Science, 38(5), 214-219.

Chenier, T. "The importance of thorough evaluation of the fetal membranes of the mare. (2011). " Equine Veterinary Education 23.3: 119-120.

Frazer, G. S. Comprometimento do cordão umbilical como causa do aborto. (2007). Equine Vet Educ; 19: 535 - 7.

Hong, C. B., Donahue, J. M., Giles, R. C, Petrites-Murphy, M. B. Jr., Poonacha, K. B., Roberts, A. W., Smith, B. J., Tramontin, R. R., Tuttle, P. A., \& Swerczek, T. W. (1993). Etiology and Pathology of Equine Placentites. Journal Veterinary Diagnostic and Investigation, 5, 55-63.

Knottenbelt, D. C, Holdstock, N., \& Madigan, J. E. (2004). Equine Neonatology Medicine and Surgery, Elsevier Science Limited, 6, $155-365$.

Laugier, C., Foucher, N., Sevin, C., Leon, A., \& Tapprest, J. (2011). A 24-Year retrospective study of equine abortion in Normandy (France). Journal of Equine Veterinary Science. 31, 116-123.

Le Blanc, M. M.; Macpherson, M.; \& Sheerin, P. (2004). Ascending Placentitis: What We Know About Pathophysiology, Diagnosis, and Treatment. Proceedings of 50th Annual Convention of the American Association of Equine Practitioners, v.50.

Madigan, J. E., \&House, J. K. (2002). Patent urachus, omphalitis, and other umbilical abnormalities. Smith BP, ed: Large animal internal medicine, (3a ed.), Mosby.

Machin, G. A, Hackerman, J., \& Gilber-Barness, E. (2000). Abnormal umbilical cord coiling is associated 296 with adverse perinatal outcomes. Pediatr Dev Pathol; 3:462-71.

Mariela, J., Iacomo, E., Lanci, A., Merlo, B., Palermo, C., Morris, L., \& Castadnetti, C. (2018) Características macroscópicas do cordão umbilical em cavalos Standardbred, Thoroughbred e Warmblood. Theriogenology 113166 e 170.

Mcauliffe, S. B., \& Knottenbelt, D. (2014). Color Atlas of Diseases and Disorders of the Horse, Elsevier Saunders, 5, $196-217$.

Mcgeady, T. A.; Quinn, P. J.; \& Fitzpatrick, E. S. (2006). Cardiovascular system. In: McGeady, T. A. Veterinary Embryology, ed. Blackwell Publishing, Oxford. 126-127.

Nyberg, D. A., Mcgaha, J. P., Pretorius, D. H., Pilu, G. (2002). The placenta, umbilical cord and membranes. Diagnostic Imaging of Fetal Anomalies, 2nd edn., Lippincott, Williams and Wilkin, Philadelphia, PA. 85-132.

Orsini, J. A. (1997). Management of umbilical hernias in the horse: treatment options and potencial complications. Equine Veterinary Education. 9, 7-10.

Paradis, M. R. (2006). Equine Neonatal Medicine, A Case-Based Approach, Elsevier Sauders, Philadelphia, PA, 12, $231-245$.

Pazinato, F. M.; Curcio, B. R.; Fernandes, C. G.; Feijó, L.; Schmith, R. A.; \&Nogueira, C. E. W. (2016). Histological features of the placenta and their relation to the gross and data from Thoroughbred mares. Pesquisa Veterinária Brasileira, 36(7), 665-670.

Pereira, A. S., Shitsuka, D. M., Parrreira, F. J., \& Shitsuka, R. (2018). Metodologia da pesquisa científica. $1^{\circ}$ Edição UBA/NTE/UFSM, Universidade de Santa Maria.

Pieszak, G. M., Gomes, G. C., \&Rodrigues, A. P. (2020). Fatores que interferem no processo de parto e nascimento: revisão interativa da literature. Research, Society and Development, 9(7). 
Research, Society and Development, v. 10, n. 1, e27710111790, 2021

(CC BY 4.0) | ISSN 2525-3409 | DOI: http://dx.doi.org/10.33448/rsd-v10i1.11790

Rossdale, P. D., \& Ricketts, S. W. (2002). Evaluation of the fetal membranes at foaling. Equine Vet Educ, 5, 78e82.

Schlafer, D. H. (2004) a. Postmortem examination of the equine placenta, fetus, and neonate: methods and interpretation of findings. In: Proceedings of the 50th Annual Convention of the American Association of Equine Practitioners, Denver, Colorado, USA, 4-8 December, 2004.American Association of Equine Practitioners (AAEP), p. 144-161.

Smith, K. C.; Blunden, A, S.; Whitwell, K. E.; Dunn, K. A.; \& Walles, A. D. (2008). A Survey of equine abortion, stillbirth and neonatal death in the UK from 1988 to 1997. Equine Vet J; 35; 496-501.

Snider, T. A. (2007). Umbilical cord torsion and coiling as a cause of dystocia and intrauterine foal loss. Equine Veterinary Education, 19(10), 532-534.

Stoneham, S., \& Munroe, A. G., In: Munroe, A. G., \& Weese, J. S. (2011). Equine Clinical Medicine Surgery, and Reproduction, Manson Publishing Ltd, c. $14,966-995$.

Sturion, T. T.; Sturion, M. A. T., Sturion, D. J.; \& Lisboa, J. N. (2013). Ultrasound evaluation of extra- and intra-abdominal umbilical structures involution in healthy Nelorecalves products of natural conception or in vitro fertilization. Pesquisa Veterinária Brasileira. vol.33 no.8 Rio de Janeiro Aug.

White, S. L., \& Huff, T. (1998). Retrospective study of surgical vs. medical management of umbilical remnant infections in neonates, Dorothy R. Havemeyer Neonatal Septicemia Workshop, Boston, 48.

Wilsher, S.; Ousey, J.; Whitwell, K.; et al. (2011). Three types of anomalous vasculature in the equine umbilical cord. Equine Veterinary Education, 23(3), 109-118.

Whitwell, K. E. (1975). Morphology and pathology of the equine umbilical cord. Journal of reproduction and fertility, Supplement 23, 599-603.

Whitwell, K. E. (1982). Investigations into fetal and neonatal losses in the horse. Vet Clin N Am Larger Anim Pract; 2, 313-3. 